\title{
Coexistence of Three Malignancies: Two Different Lung Cancers Synchronous with Lymphoma
}

\author{
Nalan OGAN, ${ }^{1}$ Ayşe BAHA, ${ }^{1}$ Egemen AKINCIOĞLU, ${ }^{2}$ Tevfik KAPLAN, ${ }^{3}$ Handan DOĞAN, ${ }^{4}$ Meral GÜLHAN, ${ }^{5}$ \\ 'Department of Chest Diseases, Ufuk University, Faculty of Medicine, Ankara-Turkey \\ ${ }^{2}$ Department of Pathology, Gülhane Education and Reseach Hospital, Ankara-Turkey \\ ${ }^{3}$ Department of Chest Surgery, Ufuk University, Faculty of Medicine, Ankara-Turkey \\ ${ }^{4}$ Department of Pathology, Ufuk University, Ankara-Turkey \\ ${ }^{5}$ Department of Chest Diseases, Hitit University, Ankara-Turkey
}

\begin{abstract}
SUMMARY
Epithelial tumors synchronous with hematologic malignancies are very rare. The hystopathologic type and stage of synchronous tumors are very important for prognosis. A 77-year-old male patient was diagnosed with lymphoma after an excisional biopsy was taken from the retroauricular region. Positron emission tomography, performed for lymphoma staging, revealed a positive solid nodule (SUVmax: 24.3) in the posterobasal segment of the right lower lobe and a negative subsolid nodule in the anterior segment of the upper lobe. Right lower lobectomy and wedge resection for subsolide nodule in the upper lobe were performed. Histopathological examination revealed mildly differentiated squamous cell carcinoma for the solid nodule and mildly differentiated adenocarcinoma with lepidic pattern for the subsolid nodule. The patient was treated for only lymphoma according to the decision of the tumor board, and his condition is stable for 1 year without any evidence of lung cancer recurrence. This case presents the treatment approach and the fact that triple synchronic malignant cases are rare in the literature.

Keywords: Adenocarcinoma; lymphoma; multidisciplinary approach; squamous cell carcinoma; synchronous tumors. Copyright $\odot$ 2017, Turkish Society for Radiation Oncology
\end{abstract}

\section{Introduction}

Multiple lung malignancies are cancers that require a harder and specialized approach for diagnosis and treatment. Their incidence has increased with the progress in imaging and other techniques. They can be synchronous or metachronous. Metachronous cases are more frequent because of the increased risk caused by antitumoral treatments.[1] Hematologic malignancies synchronous with epithelial lung malignancies are rare, as case reports in the literature. It is very important to define the type and stage of every single tumor for treatment strategy and prognosis in patients with multiple synchronous tumors. In this case report, a diffuse large B-cell lymphoma synchronous with two different (squamous and adenocarcinoma) tumors of the lung is presented because it is very rarely seen in the literature.

\section{Case Report}

A 77-year-old male patient was referred to our center after being diagnosed with a pulmonary lesion with suspicious tumoral activity. He was diagnosed with anaplastic large cell lymphoma after a retroauricular lymph node biopsy was performed in the first center. He had a history of smoking 50 packets/year. Positron emission tomography (PET) was performed for lymphoma staging, which revealed an SUVmax of 24.3 at the right lung superior segment that had a 17-mm nodule (Fig. 1). Pulmonary function test results were as follows: forced vital 


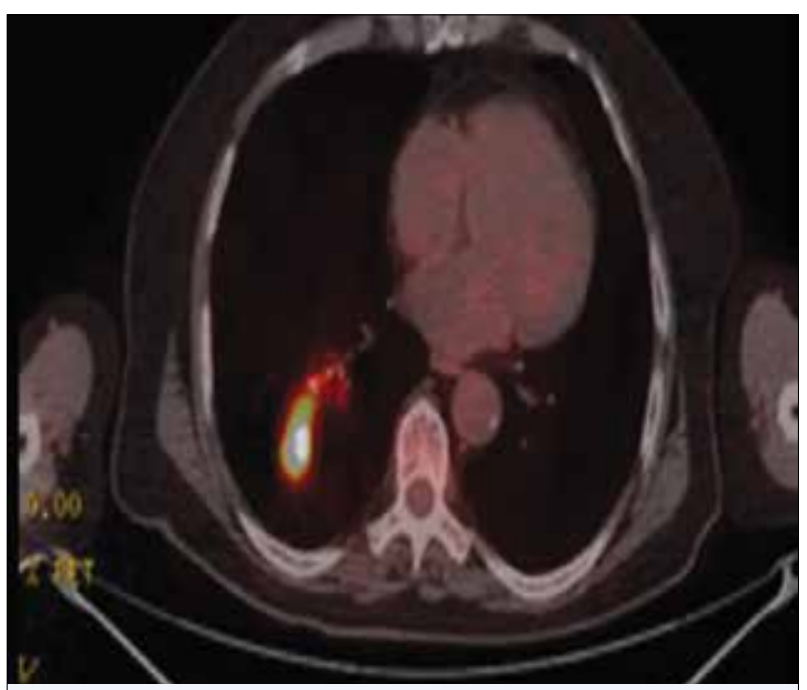

Fig. 1. Increased pathologic involvement in the $17-\mathrm{mm}$ nodule in the superior segment of the right lower lobe of the lung (SUVmax: 24.3).

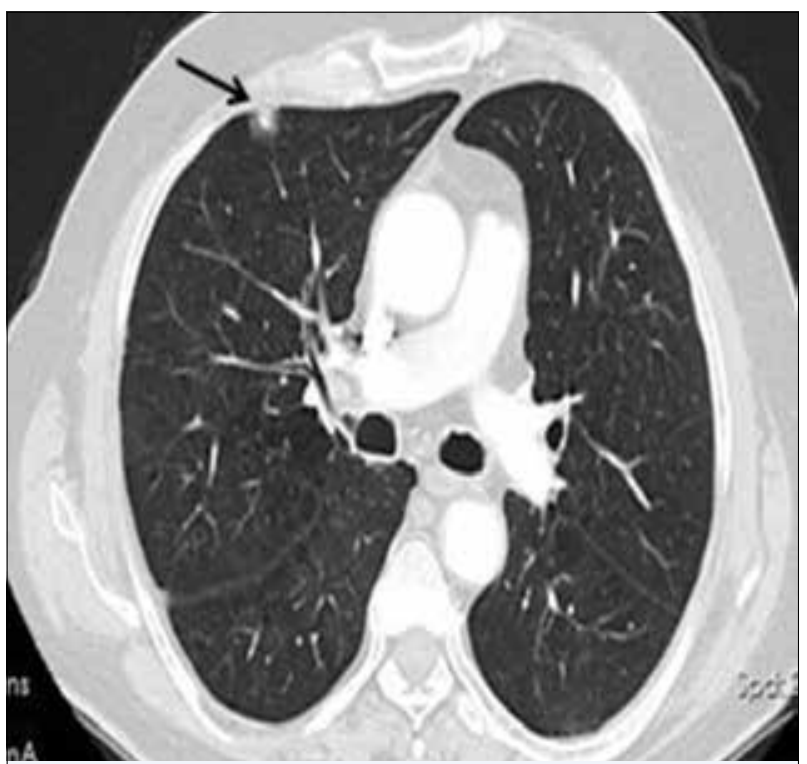

Fig. 2. A 12-mm diameter pulmonary nodule with subpleural placement in the anterior segment of the right upper lobe.

capacity (FVC): $84 \%$ (2.42 L), forced expiratory volume in the first second (FEV1): $71 \%(1.53 \mathrm{~L})$, and FEV1/FVC, 63. Chest computed tomography $(\mathrm{CT})$ revealed a nodule that had a $12-\mathrm{mm}$ circumference located in the right upper lobe anterior segment with a ground-glass density, another calcified nodule that had a 6-mm circumference at the same localization, and an additional $22 \times 18$ $\mathrm{mm}$ nodule located at the right lower lobe posterobasal segment with a spiculated contour (Fig. 2). Fiberoptic

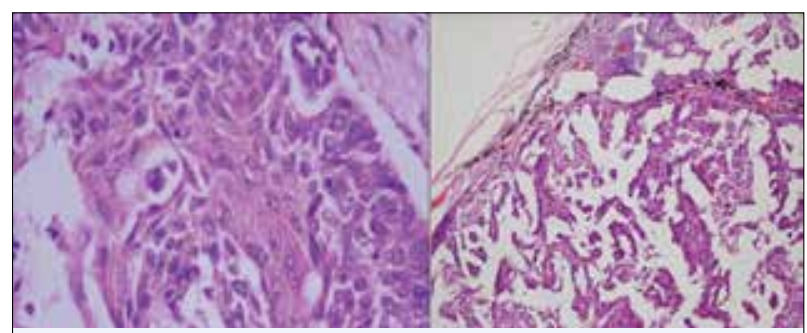

Fig. 3. A 12-mm diameter pulmonary nodule with subpleural placement in the anterior segment of the right upper lobe.

bronchoscopy revealed an irregular mucosa in the posterior segment of the right lower lobe. A biopsy result revealed squamous cell carcinoma. The patient was evaluated by the tumor board, and lung resection followed by lymphoma treatment was planned. Right lower lobectomy, upper lobe wedge resection (due to limited lung function), and mediastinal lymph node dissection were performed. Pathology results were consistent with mildly differentiated squamous cell carcinoma in the lobectomy specimen and mildly differentiated adenocarcinoma with lepidic pattern in the wedge resection specimen. There was no mediastinal lymph node metastasis. Visceral pleural invasion was detected in the lesion on the anterior segment of the upper lobe. The tumor on the lower lobe was graded as IA, whereas the tumor on the anterior segment of the upper lobe was graded as $1 \mathrm{~B}$ (Fig. 3). The patient was reevaluated by the tumor board postoperatively; adjuvant therapy was not deemed beneficial, and chemotherapy for lymphoma was planned by the medical oncology department.

\section{Discussion}

The incidence of synchronous lung cancer is $0.2 \%-20 \%$. [2] PET-CT, which has become widely used, plays an important role in the diagnosis and grading of such cases. Although rare, false negativity is possible.[3] PET scan was negative for the nodule located on the right lung upper lobe; however, our patient was diagnosed with adenocarcinoma after wedge resection. False negativity for subsolid nodules in PET-CT, like our case, is common.

Hematologic malignancy synchronous with lung cancer is very rare. Its etiology and pathophysiology is still unclear.[4] Coexistence with lung cancers in known cases is usually metachronous or diagnosed by biopsy from intrathoracic residue after lymphoma treatment. The risk of a second primary cancer increases after chemotherapy, radiotherapy, or combination therapy for malignancies with high therapeutic response such as Hodgkin's lymphoma or testicular cancer.[1] Pulmo- 
nary carcinoma synchronous with lymphoma cases are reported in the literature. We described two separate lung malignancies synchronous with lymphoma in this case report. [5,6]

Five-year survival rates of patients with synchronous tumors are lower than those with metachronous tumors. In a study by Marcel et al., patients who underwent resection for synchronous lung cancers were compared to patients with lung cancers, which had a single primary, and it was shown that approximately half of the synchronous tumors were bilateral (51\%) and 5-year survival was $23 \%$ in the early stages. A study reports that in synchronous tumors, the tumor with the most advanced stage is the best predictor for survival.[7]

Studies state that multiple pulmonary lesions have to be elucidated histopathologically because of the risk of synchronous tumors, and aggressive surgery in the early stages is necessary.[8] The American College of Chest Physicians guidelines for synchronous lung cancers state that following invasive mediastinal staging and extrathoracic imaging, every single tumor should be removed unless there is enough pulmonary reserve and no N2 involvement. Lobectomy, bilobectomy, or pneumonectomy is recommended for synchronous tumors of the same lung, while consecutive surgical resection in 4-6 weeks is recommended for bilateral synchronous tumors.[9] A study states that pneumonectomy should be avoided because of poor postoperative prognosis. [10] Every case should be evaluated according to the patient's functional status. Lobectomy for the tumor in the lower right lobe and limited (wedge) resection was performed in our case because of the patient's advanced age and comorbidities. Lesions were evaluated separately postoperatively, and adjuvant therapy for lung cancer was not planned, but lymphoma treatment without delay was performed.

\section{Conclusion}

As a result, separate diagnosis, differentiation between a metastasis and a synchronous tumor, in addition to a thorough staging is necessary for patients with multiple tumors. A multidisciplinary approach and an evaluation by a tumor board are important for the prognosis of these patients, and curative treatment for the coexistence of two different solid tumors with lymphoma was thus possible.

\section{Disclosures Statement}

The authors declare no conflicts of interest.

Ethics Committee Approval: This study was conducted inaccordance with local ethical rules.

Peer-review: Externally peer-reviewed.

\section{Conflict of Interest: None declared.}

Authorship contributions: Concept - N. O, M. G; Design A. B, T. K; Supervision - M. G; Materials - Egemen Akıncıoğlu; Handan Doğan; T. K; Data collection \&/or processing - N. O, A. B; Analysis and/or interpretation - M. G; Literature search - N. O, A. B; Writing - N. O; Critical review - M. G

\section{References}

1. van Leeuwen FE, Travis LB. Second cancers. In: DeVita VT Jr, Hellman S, Rosenberg SA, editors. Cancer: Principles and Practice of Oncology. 6th ed. Philadelphia: Lippincott Williams\&Wilkins; 2001. p. 2939-64.

2. Jung EJ, Lee JH, Jeon K, Koh WJ, Suh GY, Chung MP, et al. Treatment outcomes for patients with synchronous multiple primary non-small cell lung cancer. Lung Cancer 2011;73(2):237-42.

3. Long NM, Smith CS. Causes and imaging features of false positives and false negatives on F-PET/CT in oncologic imaging. Insights Imaging 2011;2(6):679-98.

4. Ichihara E, Tabata M, Takigawa N, Sato Y, Kondo E, Aoe $\mathrm{M}$, et al. Synchronous pulmonary MALT lymphoma and pulmonary adenocarcinoma after metachronous gastric MALT lymphoma and gastric adenocarcinoma. J Thorac Oncol 2008;3(11):1362-3.

5. Rothenburger M, Semik M, Hoffmeier A, Baba H, Kamanabrou D, Roos N, et al. Coexistence of nonHodgkin's lymphoma and non-small cell lung carcinoma: diagnosis and treatment. Thorac Cardiovasc Surg 2002;50(1):59-61.

6. Zheng JX, Li XZ, Xiang RL, Gao Z. Synchronous primary pulmonary lymphoma presenting with pulmonary adenocarcinoma: A case report and literature review. Indian J Cancer 2015;52 Suppl 1:e37-40.

7. van Rens MT, Zanen P, Brutel de La Rivière A, Elbers HR, van Swieten HA, van Den Bosch JM. Survival in synchronous vs. single lung cancer: upstaging better reflects prognosis. Chest 2000;118(4):952-8.

8. Uçgun I, Akçayir Sahin I, Metintaş M, Alataş F, Erginel S, Dündar E. Synchronous primary lung cancers: due to the four cases. Tuberk Toraks 2004;52:262-7.

9. Detterbeck FC, Postmus PE, Tanoue LT. The stage classification of lung cancer: Diagnosis and management of lung cancer, 3rd ed: American College of Chest Physicians evidence-based clinical practice guidelines. Chest 2013;143(5 Suppl):e191S-e210S.

10. Liu M, He W, Yang J, Jiang G. Surgical treatment of synchronous multiple primary lung cancers: a retrospective analysis of 122 patients. J Thorac Dis 2016;8(6):1197204. 